\title{
Agôn
}

Revue des arts de la scène

Critiques | Saison 2017-2018

\section{Finir en Beauté, quelle historiographie du deuil ?}

\section{Camille Khoury}

\section{OpenEdition}

Journals

Édition électronique

URL : http://journals.openedition.org/agon/4395

DOI : 10.4000/agon.4395

ISSN : 1961-8581

\section{Éditeur}

Association Agôn

\section{Référence électronique}

Camille Khoury, "Finir en Beauté, quelle historiographie du deuil ? », Agôn [En ligne], Critiques, mis en ligne le 22 mai 2018, consulté le 26 avril 2019. URL : http://journals.openedition.org/agon/4395 ; DOI : 10.4000 /agon.4395

Ce document a été généré automatiquement le 26 avril 2019

Association Agôn et les auteurs des articles 


\title{
Finir en Beauté, quelle historiographie du deuil ?
}

\author{
Camille Khoury
}

\section{RÉFÉRENCE}

Finir en Beauté - spectacle de Mohamed El Khatib créé en 2014, représenté le 18 mai 2018, Théâtre Garonne, Toulouse

1 Programmé au théâtre Garonne dans le cadre du festival d'histoire L'Histoire à venir, le spectacle Finir en Beauté, crée en 2014 à Marseille dans le cadre du festival actOral, nous propose un parcours documenté dans une tranche de la vie de son auteur, Mohamed El Khatib, qui retrace pas à pas le décès de sa mère. Pour cela, le metteur en scène et interprète a installé un cadre intimiste, où le public est installé sur la scène en arc de cercle, et prend place sur des petits tabourets en bois placés face à un dispositif scénique simplement constitué d'une table et d'une télévision.

2 S'il nous fait entrer dans le spectacle par la fiction, en commençant par nous lire l'extrait de sa première pièce, dans laquelle il imagine la place idéale du fils accompagnant la mort de sa mère, Mohamed El Khatib annonce dès la fin de cette lecture que le réel n'a pas suivi la fiction, et que c'est au récit du réel plutôt qu'au récit fictif qu'il compte ici s'attacher. Pour partir en quête du réel, le metteur en scène est allé chercher des traces, des documents de tous formats (photographies, enregistrements sonores, actes de naissances et actes de décès, carnets de notes et journaux intimes, etc.), qu'il nous expose les uns après les autres, racontant parfois une anecdote, refaisant pas à pas les étapes du décès, du moment où il apprend que sa mère est condamnée à son enterrement au Maroc.

3 En retraçant les événements non pas à partir de ses seuls souvenirs mais en documentant le réel, Mohamed El Khatib n'est pas autobiographe : il ne nous raconte pas un moment de sa vie, il offre le produit d'une enquête sur son passé, à la recherche de traces d'un moment révolu, pour permettre d'en reconstituer des faits qu'il a lui-même vécus. En un mot, il se fait l'historien de sa propre histoire. 
Plus tôt dans la journée, dans la grande salle du Théâtre Garonne, l'historien Johann Chapoutot avait proposé une conférence sur l'épistémologie de l'histoire, cette science sociale si particulière où l'humain s'interroge sur lui-même. Il exposait l'évolution des conceptions du rôle de l'historien et les différentes formes qu'a pris l'historiographie dans le temps. Aujourd'hui, et depuis l'école des Annales, l'historien n'est plus, contrairement à l'historien de l'Antiquité, ce collecteur du réel, ce «fouilleur de déchets ", qui cherche dans les poubelles du temps des traces du passé qu'il se contente de défroisser et de ramener en surface. Le travail de l'historien ne consiste pas simplement en l'exhibition de ses documents, il s'interroge sur leur signification, sachant qu'un document n'est jamais un morceau de réel mais un éclat partial et partiel. Il s'agit alors d'en déterrer les partis pris, l'utilité, le contexte, de le mettre en lien avec d'autres documents non pas en vue d'établir une collection, mais de produire du sens.

5 Au cours de cette archéologie personnelle, Mohamed El Khatib prend le parti de la distance de l'exposé plutôt que du récit subjectif. Dans son jeu sur scène, il use du ton neutre de celui qui parle en public, qui fait un exposé ou un cours, cette parole construisant une neutralité, mettant à distance les émotions du locuteur. Il expose un moi issu du passé qu'il n'incarne pas, et cette utilisation du document, comme de la position qu'il occupe par rapport à lui, produit un sentiment de malaise.

6 En mettant en avant les archives du décès plutôt que sa propre subjectivité au sein des événements, Mohamed El Khatib semble nous raconter une histoire qui n'est plus sienne mais celle de quelqu'un d'autre, à laquelle il a assisté, comme témoin extérieur. Il ne fait pas l'histoire de son deuil, mais celle d'un décès, partage le corps malade et mourant de sa mère en nous obligeant à un voyeurisme auquel ni elle ni nous n'avons consenti. Cette impression de voyeurisme, déjà très présente lorsque l'on reçoit les documents qu'il nous donne à voir - les enregistrements sonores de conversations avec sa mère défunte, la photocopie de l'acte de décès (qui donne à la pièce son sous-titre : Pièce en un acte de décès), la photographie du corps défunt de sa mère - est d'autant plus présente que les documents appartiennent à la vie privée d'une famille.

7 Je discutais récemment avec une amie qui me disait que selon elle, l'intime est partageable, et le privé est justement ce que l'on ne rend pas public. Le spectacle de Mohamed El Khatib m'aurait sans doute mise moins mal à l'aise s'il avait rendu la trace intime du deuil partageable plutôt en exhibant un espace privé simplement utilisé comme matériau, sans les rendre signifiants autrement que dans leur monstration. À force de se mettre à distance et de s'effacer de sa propre histoire, il met en place un dispositif voyeuriste fondé sur la croyance selon laquelle le document comme trace objective d'histoire se suffit à lui-même, et déplace l'objet du spectacle de son deuil à la mort de sa mère.

8 Malgré sa maladresse, on sent dans ce spectacle un hommage, à l'image de celui qu'Albert Cohen a voulu offrir à sa propre mère en écrivant son Livre sur ma mère. Tout comme Albert Cohen, qui met la judéité familiale comme point d'entrée de son rapport à sa mère, Mohamed El Khatib met en lumière à quel point l'ancrage marocain de sa culture familiale a été important dans ces événements, dans le rapport à la mère, à la mort, la barrière de la langue, la communication avec les médecins, le transport du corps au Maroc pour l'enterrement. On sent clairement l'émotion affleurer lors de ces évocations. Mais il faut attendre la dernière image du spectacle pour que Mohamed El Khatib s'incarne enfin. Debout devant la tombe de sa mère, il laisse place brièvement à la douleur de la perte. 